\title{
A high throughput system in vitro to evaluate the effects of fruits to modulate the inflammation biomarker $\mathrm{NF \kappa} \beta$
}

\author{
L. Tomás-Cobos ${ }^{1}$, A. Rodrigo ${ }^{1}$, D. Forbes ${ }^{2}$, J. Zhang ${ }^{2}$ and M. Skinner ${ }^{2}$ \\ ${ }^{1}$ Ainia Technological Centre, Parque Tecnológico de Valencia, c. Benjamín Franklin 5-11 E 46980 Paterna (Valencia) and \\ ${ }^{2}$ New Zealand Institute for Plant and Food Research Ltd, Private Bag 92169, Auckland 1142, New Zealand
}

Growing interest for wellness and healthy natural food products has impacted on the food market with the development of new products with healthy properties beyond basic nutrition, known as functional foods ${ }^{(1)}$. A common strategy for functional food development is based on adding ingredients containing biocompounds with a known beneficial activity. An alternative to designing specific functional products is to use natural food extracts as a source of bioactive compounds ${ }^{(2,3)}$. One of the health targets for functional foods is inflammation, an essential response of the immune system to infection and damaged tissue. However, uncontrolled inflammation processes can lead to tissue damage and chronic diseases ${ }^{(4,5)}$. At the molecular level, it is considered that the transcriptional nuclear factor $\mathrm{NF \kappa} \beta$ plays a central role to control the expression of pro-inflammatory mediators such as interleukins (ILs), for example IL-1, IL-6 and IL-8, and tumour necrosis factor $\alpha$ (TNF- $\alpha)^{(6,7)}$.

The aim of the present study was to investigate whether fruit extract mixtures could modulate inflammation in vitro.

A high throughput in vitro assay was used, where the expression of $\mathrm{NF \kappa} \beta$ was evaluated in the presence of two fruit extracts (Ffowcs Williams Ltd, Auckland, New Zealand) and mixtures of them to determine whether any synergistic interactions between the extracts could be determined. Orange and cranberry were the studied fruits. Both are rich in bioactive compounds, orange as a source of bioflavonoids and cranberry as a source of anthrocyanidins. The NF- $\mathrm{KB}$ assay involved transfecting HEK-293 (human embryonic kidney) cells with a plasmid containing NFK $\beta$ promoter elements linked to a secreted embryonic alkaline phosphatase (SEAP) reporter system (pNiFty-SEAP; InvivoGen, San Diego, USA). Transfected cells were incubated with purified orange and cranberry extracts and combinations of them based on their total phenolic contents (expressed as catechin equivalents). A stress was induced by adding $10 \mu \mathrm{M}$ hydrogen peroxide $\left(\mathrm{H}_{2} \mathrm{O}_{2}\right)$. After incubation, the cell culture supernatants were collected to analyse SEAP activity and cytotoxicity. To determinate levels of SEAP expression, we used Quanti-Blue reagent according to the manufacture's instructions (InvivoGen, San Diego, USA). To determinate cytotoxicity, we used Alamar Blue cell cytotoxicity assay ${ }^{(8)}$.

At concentrations of orange and cranberry extract used here were not cytotoxic to the HEK-293 cells (data not shown). The results obtained indicate that both the orange and cranberry extracts and mixtures of them exhibited anti-inflammatory activity by their ability to inhibit NFK $\beta$ expression with this activity being dependent on the concentration and ratio of each fruit extract. We observed an additive effect at $9 \mu \mathrm{gg} / \mathrm{ml}$ catechin equivalent. Although the cranberry extract had little effect at this dose, addition of an increasing ratio of the orange extract to the cranberry extract decreased the activation of NFK $\beta$ in a ratio-dependent manner (Fig. 1). At a concentration $37 \mu \mathrm{gg} / \mathrm{ml}$ catechin equivalent, we observed a synergistic effect. Mixtures of the two fruit extract decreased the activation of NFK $\beta$ more than either fruit extract alone (Fig. 2).

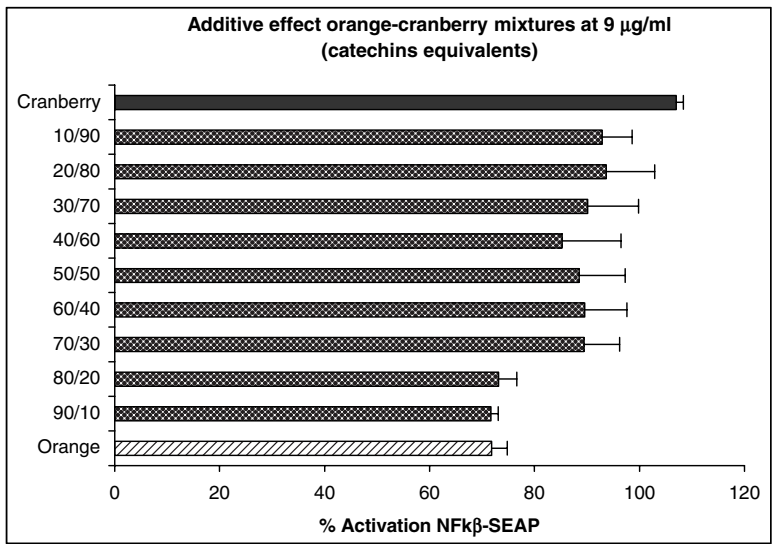

Fig. 1. Additive effects of orange-cranberry extract mixtures on HEK-293

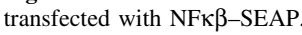

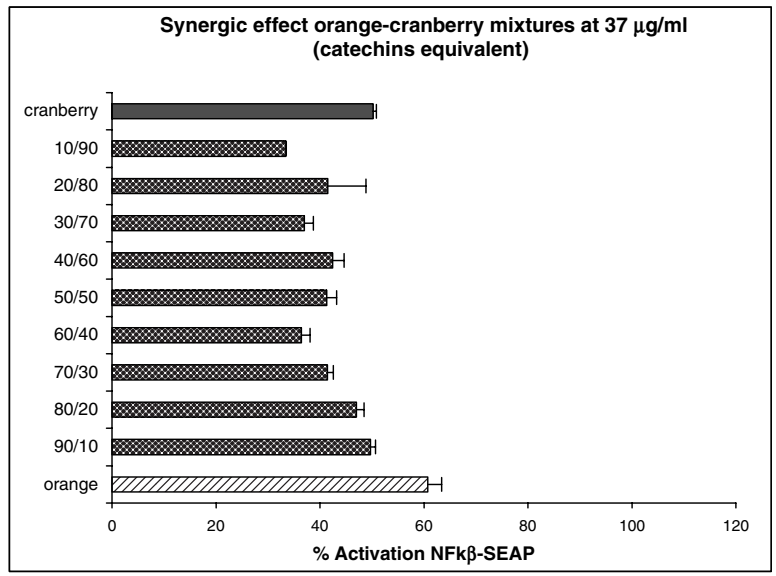

Fig. 2. Synergic effects of orange-cranberry extract mixtures on HEK-293 transfected with NFK $\beta$-SEAP.

It is probable that the health benefits of fruits and vegetables are based on complex interactions between their components. It is important to determine these interactions and the best combinations to use to develop functional foods. The results of this study demonstrate that a high throughput in vitro assay system based on NFK $\beta$ activity to screen for anti-inflammatory or pro-inflammatory effects is a useful tool to study the interaction of food extracts or ingredients. The data obtained from such screening provide the support required to proceed to testing novel functional foods in animal models and human intervention trials.

This study has been developed with the support of IMPIVA (Instituto de la Mediana y Pequeña Empresa valenciana)-EUROPEAN SOCIAL FUND through the Programme of Development of Human Potential in the field of Research, Technological development and innovation 2008-2009.

1. Hasler CM (1998) Food Tech 52, 63-70.

2. Nassiri-Asl M \& Hosseinzadeh H (2009) Phytother Res 2009 Jan 12 [Epub ahead of print].

3. Benavente-Garcia O \& Castillo J (2008) J Agric Food Chem 56, 6185-6205.

4. Calder PC (2006) Scand J Food Nutr 50, 54-61.

5. Grimble RF (1998) Nutrition 14, 634-640.

6. Shoelson SE, Lee J \& Yuan M (2003) J Obes Relat Metab Disord 3, 49-52.

7. Aggarwal BB (2004) Cancer Cell 6, 203-208.

8. Wilson OI, Orton T \& Pognan F (2000) Eur J Biochem 267, 5421-5426. 